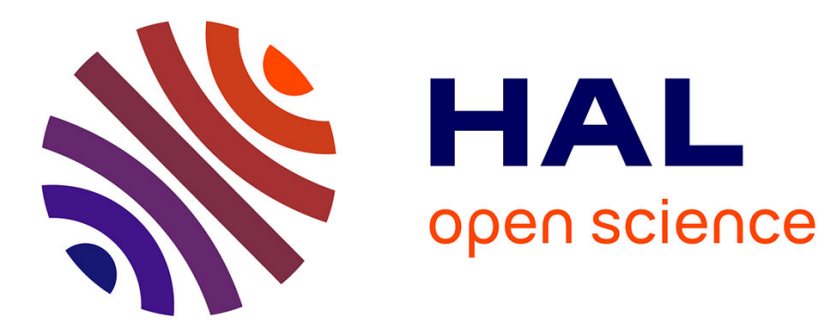

\title{
Shear strength of unsaturated soils: experiments, DEM simulations, and micromechanical analysis
}

Vincent Richefeu, Moulay Saïd El Youssoufi, Farhang Radjai

\section{To cite this version:}

Vincent Richefeu, Moulay Saïd El Youssoufi, Farhang Radjai. Shear strength of unsaturated soils: experiments, DEM simulations, and micromechanical analysis. 2nd International Conference on Mechanics of unsaturated soils, Mar 2007, Weimar, Germany. pp.83-91. hal-00147621

\section{HAL Id: hal-00147621 \\ https://hal.science/hal-00147621}

Submitted on 18 May 2007

HAL is a multi-disciplinary open access archive for the deposit and dissemination of scientific research documents, whether they are published or not. The documents may come from teaching and research institutions in France or abroad, or from public or private research centers.
L'archive ouverte pluridisciplinaire HAL, est destinée au dépôt et à la diffusion de documents scientifiques de niveau recherche, publiés ou non, émanant des établissements d'enseignement et de recherche français ou étrangers, des laboratoires publics ou privés. 


\title{
Shear Strength of Unsaturated Soils: Experiments, DEM Simulations, and Micromechanical Analysis
}

\author{
Vincent Richefeu ${ }^{1}$, Moulay Saïd El Youssoufi ${ }^{2 *}$, and Farhang Radjaï ${ }^{3}$ \\ LMGC UMR CNRS 5508, Université Montpellier 2, Place Eugène Bataillon \\ 34095 Monpellier Cedex 5, France \\ ${ }^{1}$ richefeu@lmgc .univ-montp2.fr, \\ $2 *$ elyous@lmgc .univ-montp2.fr, \\ 3 radjai@lmgc.univ-montp2.fr
}

\section{Introduction}

The interest of scientists for granular materials is motivated by the important place they occupy in our natural environment. Well-known examples are soils, rocks and powders. In addition, many industrial processes implement transformations of granular materials such as pharmaceutical tablets, agricultural and food products, building materials (Bika et al., 2001; Iveson et al., 2002). The mechanical behavior of these materials, in particular soils, was widely developed in the context of macroscopic continuum approach. This approach is based on the concept of Representative Elementary Volume (REV) which is not well-established for granular media. Moreover, it introduces many parameters that require a large number of identification experiments. In addition, most continuum approaches do not account for grain-scale mechanisms such as the initiation and propagation of shear bands or cracks.

On the other hand, the discrete character of granular materials makes it possible to identify unambiguously the grain scale and thus the proper kinematics and mechanical behavior at this scale. Grain-scale properties depend on composition, form, size or surface state of grains. A discrete approach requires furthermore a rigorous definition of the interactions of each grain with its close neighbors through mechanical contacts, friction and/or cohesion. External actions like the moisture, temperature and pressure can also act on the evolution of these interactions. This is a promising approach for understanding all the complexity and often surprising properties of granular materials if adequate techniques can be developed. Such techniques are often referred to as Discrete Elements Method (DEM). This method has been successfully applied to study classical problems in soil mechanics as shear bands localization (Bardet and Proubet, 1991), slope stability (Staron et al., 2002) and

Page: 1 job:Richefeu macro: svmult.cls date/time: 18-0ct-2006/20:26 
shear strength (Jiang et al., 2004; Thornton and Antony, 2000; Richefeu et al., 2005).

In this paper, we investigate shear strength properties of wet granular materials as a function of water content in the pendular state. Sand and glass beads were wetted and tested in a direct shear cell. In parallel, we carried out DEM simulations by means of a 3D numerical code (tapio-K) based on molecular dynamics approach developed in our laboratory. We used an explicit expression of the capillary force as a function of interparticle distance, water bridge volume and surface tension. A peculiar feature of our system is that the effects of capillary bonding can be observed only if the confining pressure is of the same order of magnitude or below the tensile strength or the Coulomb cohesion. The latter is proportional to the strength of capillary bonds and inversely proportional to the particle size (Richefeu et al., 2006). For fine sand, typical values are below $1 \mathrm{kPa}$, imposing us to work with low confining pressure.

We find good agreement between experiments and numerical simulations. We show that the shear strength is mostly controlled by the distribution of liquid bonds. This property results in the saturation of shear strength as a function of water content. We arrive at the same conclusion by analyzing the shear strength from the microstructure and by accounting for grain polydispersity. Finally, we discuss the applicability of the discrete element approach to unsaturated soils under more general loading paths.

\section{Discrete Element Method}

We developed a DEM code based on the Molecular Dynamics (MD) method (Cundall and Strack, 1979; Allen and Tildesley, 1987). The grain motions are explicitly integrated by accounting for their interactions (e.g., contact, friction, capillary cohesion, magnetisation ....). Here, we present the interaction laws used for the contact, friction and capillary cohesion in the case of spherical grains.

\subsection{Contact and Friction Laws}

The normal contact law is modeled as a spring and a dashpot in parallel. The normal force $f_{n}^{c t c}$ is given by

$$
f_{n}^{c t c}=-k_{n} \delta_{n}+\gamma_{n} v_{n}
$$

where $\delta_{n}$ is the normal distance (negative or zero when the grains are in contact), $k_{n}$ is the normal stiffness, $v_{n}$ is the relative normal velocity, and $\gamma_{n}$ is a damping coefficient.

The friction law expresses the tangential force as a function of the sliding velocity $\mathbf{v}_{s}$. The friction force $\mathbf{f}_{t}$, contained in the contact plane, obeys a viscous-regularized Coulomb law:

Page: 2 job: Richefeu macro: svmult.cls date/time: 18-0ct-2006/20:26 


$$
\mathbf{f}_{t}=\min \left\{\gamma_{t} \mathbf{v}_{s}, \mu f_{n}^{c t c}\right\} \frac{\mathbf{v}_{s}}{\left\|\mathbf{v}_{s}\right\|},
$$

where $\gamma_{t}$ is the tangential viscosity and $\mu$ is the coefficient of friction.

\subsection{Capillary Cohesion Law}

In the presence of water, the grains can be connected by a liquid bridge as illustrated in Fig. 1a. The liquid bridge gives rise to an attractive force, the force of capillary cohesion, between the grains. This force results from the combined action of the difference in pressure between the liquid and gas phases and the liquid surface tension $\gamma$ (Pierrat and Caram, 1997; Willett et al., 2000; Gröger et al., 2003; Soulié et al., 2006). We use the explicit expression of the capillary law of cohesion proposed by Soulié et al. (2006) in the polydisperse case (Fig. 1b). This law, expressing the capillary force $f_{c}$ as a function of the normal distance $\delta_{n}$, is given by

$$
f_{c}= \begin{cases}-\pi \gamma \sqrt{R_{1} R_{2}}\left\{\exp \left(A \frac{\delta_{n}}{R_{2}}+B\right)+C\right\} & \text { for } \delta_{n}>0 \\ -\pi \gamma \sqrt{R_{1} R_{2}}\{\exp (B)+C\} & \text { for } \delta_{n} \leq 0\end{cases}
$$

where $R_{1}$ and $R_{2}$ are the grains radii $\left(R_{1}<R_{2}\right) ; A, B$ and $C$ are parameters which depend on the volume $V_{b}$ of the liquid bond and on the contact angle $\theta$; $\delta_{0}$ is the rupture distance. From a theoretical study of a liquid bridge between two spheres, Lian et al. (1993) proposed the following rupture criterion:

$$
\delta_{0}=(1+0.5 \theta) V_{b}^{1 / 3} .
$$

We used this criterion which was validated by local experiments (Soulié, 2005). It is also necessary to define a criterion for the formation of a liquid bond. It is considered that a liquid bridge can be formed (or to reform itself) when the grains are in contact $\left(\delta_{n} \leq 0\right)$. At the numerical level, the main difficulty comes
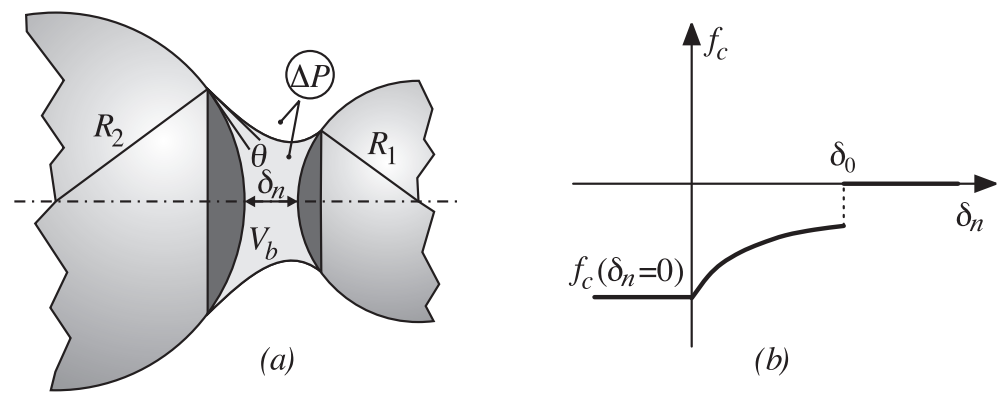

Fig. 1. Capillary cohesion law. (a) Physical and geometric parameters of a capillary bond. (b) Typical behavior of the capillary strength as a function of the normal distance

Page: 3 job:Richefeu macro: svmult.cls date/time: 18-0ct-2006/20:26 
from the management of the distribution of the total volume of water between the grains, i.e. the determination of local volumes of the capillary bonds. In the simulations presented in this paper, the total volume of water is distributed over all the possible bonds of the sample in proportion to the radii of the touching grains. This corresponds to a global and homogeneous management of the distribution of water, which makes it possible to connect the local water volumes to the macroscopic water content. Local water redistributions were considered, too, see Richefeu (2005). The distribution protocol plays a determining role in the mechanical resistance of material, as we shall see below. However, this information remains difficult to reach in experiments with real materials.

\section{Direct Shearing at Low Confining Stresses}

Several modifications were made to the standard Casagrande's shear box in order to be able to measure the shear strength of granular materials to very low confining pressures (lower than $1 \mathrm{kPa}$ ). We present here the main experimental and numerical results obtained. These results will be analyzed through a theoretical expression of the Coulomb cohesion resulting from a microscopic analysis.

\subsection{Experiments and Simulations}

Figure 2 shows a schematic view of the setup, its dimensions, and certain notations used. A normal pressure $\sigma=g\left(m_{N} / S+\rho h\right)$ is applied to the sample by means of a flask whose mass $m_{N}$ can be modified by pouring sand into it. $\rho$ is the bulk density and $g$ is the gravity. A shear stress $\tau=m_{T} / S$ is incrementally imposed by gradually filling a cup connected to the moving part of the box by a system of pulley. The stress reached during the rupture of the sample is the yield shear stress. Several tests carried out with various normal stresses allow us to estimate the yield locus in the Mohr-Coulomb plane. In direct shearing, the boundary conditions are such that the finite size effects cannot be fully eliminated (Thornton and Zhang, 2001). We did not check such size effects in our experiments. Given the system size, this requires using considerably more particles with smaller size, affecting thus the level of Coulomb cohesion in the presence of water. But we checked that the measured strengths are reproducible and in good agreement with analytical estimates (see below).

Figure 3a shows the yield loci obtained for a sand made up of angular grains of diameters ranging from 0.1 to $0.4 \mathrm{~mm}$ with various water contents $w$ (ratio of the water mass on grains mass). One can notice that the experimental points are well fit to straight lines, in agreement with the Mohr-Coulomb model $\tau=\tan \varphi \sigma+c$ characterized by two parameters: the macroscopic cohesion $c$ and the internal friction angle $\varphi$. This figure also shows the good

Page: 4 job: Richefeu macro: svmult.cls date/time: 18-0ct-2006/20:26 


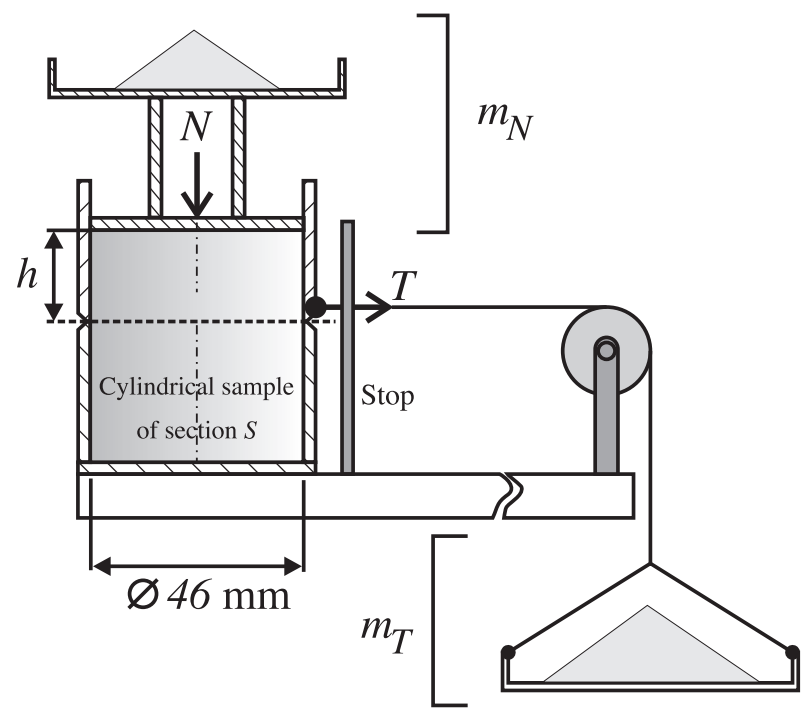

Fig. 2. Experimental Setup

reproducibility of the experiments. The angle $\varphi$ does not seem to vary appreciably with $w$ in contrast to the parameter of cohesion which increases in a nonlinear way with water content and is saturated to a value $c_{m}$ for a water content $w_{m}$ (Fig. 3b). For the water contents above $5 \%$, we leave the pendular regime.

The numerical tests are similar to the experimental tests (same level of loading, same dimensions of the box ... ). A numerical sample of 7307 spherical
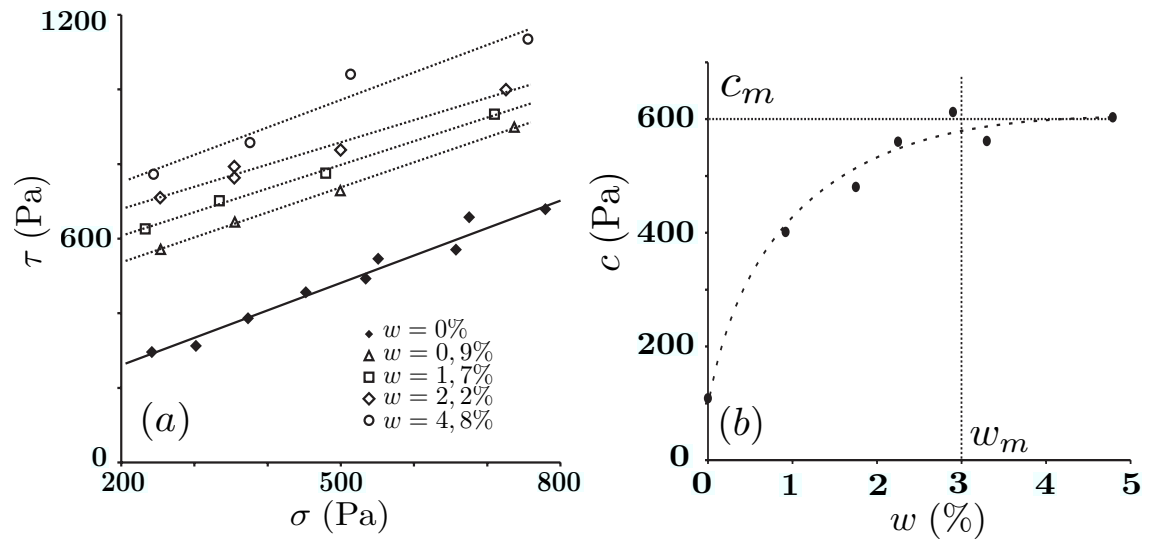

Fig. 3. Experimental results. (a) Yield loci estimated for a sand. (b) Coulomb cohesion as a function of water content 


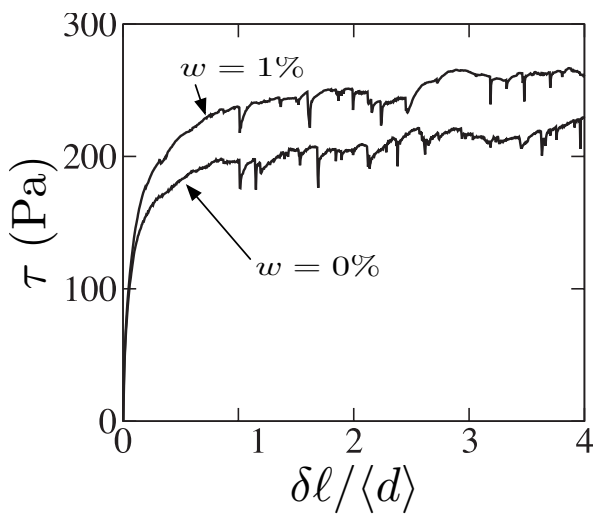

Fig. 4. Stress-strain relations for $w=0 \%$ and $w=1 \%(\sigma=300 \mathrm{~Pa})$

grains was prepared with a solid fraction $\phi=0.6$. The number of particles is imposed by the system dimensions and particle size in order to reach the same level of packing fraction as in experiments. Various water contents were obtained by distributing the total water volume over all the contacts $\left(\delta_{n} \leq 0\right)$ in this sample. A set of 15 simulations (three levels of the normal stress and five values of the water content) were performed. Figure 4 presents the stressstrain plots obtained for $w=0 \%$ and $w=1 \%$. The rupture stress is considered to be the residual shear stress because the shear stress approaches the residual state without passing by a peak state. As for the experiments, the evolution of the macroscopic cohesion can be analyzed as a function of the water content. Figure $5 \mathbf{a}$ shows the estimates of yield loci obtained numerically. One can note that the numerical model reproduces well the experimental data. The water content does not have an influence on the internal angle of friction and the cohesion parameters increases in a nonlinear way and saturates at a relatively low level of the water content (Fig. 5b).

\subsection{Micromecanical Analysis}

From a micromechanical analysis, a theoretical expression of the Coulomb cohesion was established (Richefeu et al., 2006):

$$
c^{t h}=\frac{3}{2 \pi} \mu \kappa s \frac{\phi z}{\langle D\rangle},
$$

where $\kappa$ is a parameter that depends essentially on the surface tension of water, $s$ is a factor related to the polydispersity of material, $z$ is the average number of liquid bonds per grain, and $\langle D\rangle$ is the average diameter of the grains. The validity of this expression was shown by comparing the predicted theoretical values of Coulomb cohesion with experimental and numerical measurements (Table 1). This comparison shows also that the numerical results are in quantitative agreement with the experiments. 

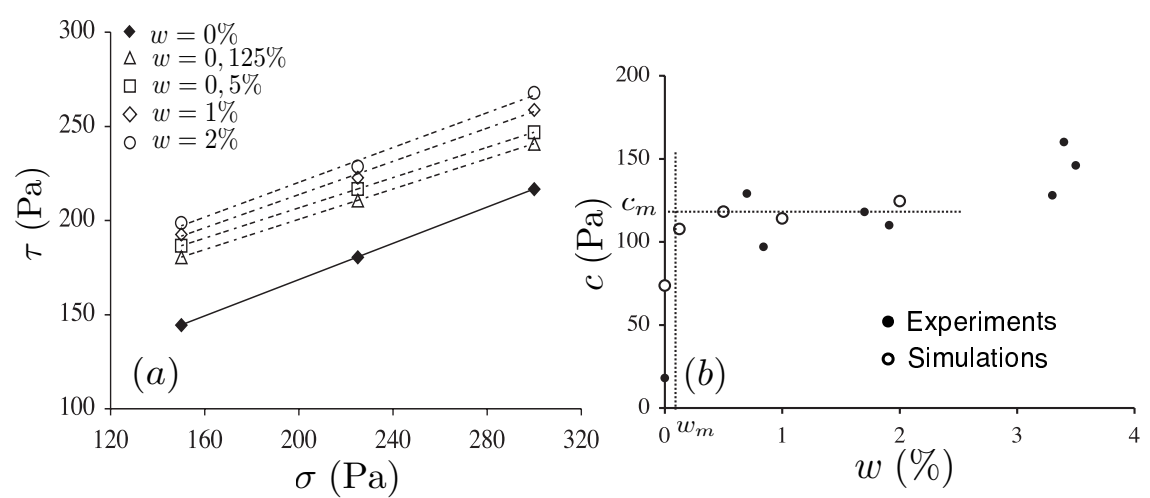

Fig. 5. Simulation results. (a) Estimated yield loci. (b) Coulomb cohesion as a function of water content (experiments were carried out with $1 \mathrm{~mm}$ glass beads)

Table 1. Measured and theoretical parameters for the experimental and numerical samples

\begin{tabular}{llllll}
\hline & Sand & GB1 & GB2 & GB3 & Simulations \\
\hline$\langle D\rangle(\mathrm{mm})$ & 0.16 & 0.45 & 0.60 & 1.00 & 1.65 \\
$s$ & 0.50 & 0.99 & 0.91 & 1.00 & 0.79 \\
$z$ & 6 & 6 & 6 & 6 & 9 \\
$\phi$ & 0.6 & 0.6 & 0.6 & 0.6 & 0.6 \\
$\tan \varphi$ & 0.66 & 0.58 & 0.58 & 0.46 & 0.48 \\
$c_{m}(\mathrm{~Pa})$ & 600 & 350 & 300 & 150 & 120 \\
$c_{t h}(\mathrm{~Pa})$ & 709 & 438 & 302 & 158 & 118 \\
\hline
\end{tabular}

In Table 1, we show also experimental results of three other materials (Richefeu et al., 2006; Richefeu, 2005): (1) tightly-graded polydisperse glass beads with diameters from $0.4 \mathrm{~mm}$ to $0.5 \mathrm{~mm}$ (denoted by GB1), (2) wellgraded polydisperse glass beads with diameters from $0.4 \mathrm{~mm}$ to $0.8 \mathrm{~mm}$ (denoted by GB2), and (3) monodisperse glass beads of diameter $1 \mathrm{~mm}$ (denoted by GB3).

\section{Conclusions}

Experiments and the simulations based on discrete element simulations were used with the aim of studying and analyzing the shear strength of unsaturated granular materials in the pendular state at low levels of loading. It was shown that the Coulomb cohesion increases with the water content and that it is saturated to a maximum value which depends only on the nature of the material. A theoretical analysis based on micromechanics allows us, on one hand, to validate the numerical modeling and, on the other hand, to show

Page: $7 \quad$ job: Richefeu macro: svmult.cls date/time: 18-0ct-2006/20:26 
that the increase in the shear strength with water content is primarily due to an increase in the number of liquid bonds per unit volume.

In the extension of this work, it is essential to evaluate the limits of the model by considering other materials and non monotonous loading paths. In particular, we would like to study the shear strength of granular media with a larger polydispersity than materials that were used in the present investigation. Since the distribution of liquid bonds seems to be a major parameter for the cohesion of unsaturated granular materials, it also merits to be investigated in more detail experimentally. Finally, an interesting application of the ideas put forward in this paper would be to examine by which mechanisms the cohesion of a sample of unsaturated sand increases as a result of compactification.

\section{References}

Allen MP, Tildesley DJ (1987) Computer Simulation of Liquids. Oxford University Press, Oxford

Bardet JP, Proubet J (1991) Geotechnique 41(4):599-613

Bika D, Gentzler M, Michaels J (2001) Powder Technology 117:98-112

Cundall PA, Strack ODL (1979) Geotechnique 29:47-65

Gröger T, Tüzün U, Heyes DM (2003) Powder Technology 133:203-215

Iveson S, Beathe J, Page N (2002) Powder Technology 127:149-161

Jiang MJ, Leroueil S, Konrad JM (2004) Computers and Geotechnics 31:473-489

Lian G, Thornton C, Adams MJ (1993) Journal of Colloid and Interface Science 161:138-147

Pierrat P, Caram H (1997) Powder Technology 91:83-93

Richefeu V (2005) Approche par éléments discrets 3D du comportement de matériaux granulaires cohésifs faiblement contraints. PhD Thesis, Université Montpellier 2, France

Richefeu V, El Youssoufi MS, Peyroux R, Bohatier C (2005) Frictional contact and cohesion laws for Casagrande's shear test on granular materials by 3D DEM comparison with experiments. In: García-Rojo R, Herrmann HJ, McNamara S (eds) Powders and Grains 2005. A.A. Balkema Publisher, pp. 509-512.

Richefeu V, El Youssoufi MS, Radjaï F (2006) Physical Review E 73:051304

Soulié F (2005) Cohésion par capillarité et comportement mécanique de milieux granulaires. PhD Thesis, Université Montpellier 2, France

Soulié F, Cherblanc F, El Youssoufi MS, Saix C (2006) Int J Numer Anal Meth Geomech 30:213-228

Staron L, Vilotte JP, Radjaï F (2002) Physical Review Letter 89(20):204302

Thornton C, Antony SJ (2000) Powder Technology 109:179-191

Thornton C, Zhang L (2001) A DEM comparison of different shear testing devices. In: Kishino (ed) Powders and Grains 2001. A.A. Balkema Publisher, pp. 183-190

Willett C, Adans MJ, Johnson S, Seville J (2000) Langmuir 16:9396-9405 